\title{
Protocollo per la prevenzione delle CRBSI
}

\author{
Antonella Montani, Lucia Pozzi, Lucia Granatieri, Mauro Magugliani, Ornella Fusè, \\ Marzia Moretti, C.I.
}

Reparto di Nefrologia e Dialisi, Azienda Ospedaliera Legnano, Presidio Magenta, Legnano (MI)

\begin{abstract}
Prevention of Catheter-Related infections
Abstract. Since 1993 in our dialysis center we adopted a protocol for the prevention of catheter-related infections. We hereby describe it by emphasizing the very low infection rates that we recorded in the last 10 years: below 1/1,000 days of catheter permanence.
\end{abstract}

Key words: Protocol, Hemodialysis, Catheter-related infections (CRBSI)

Conflict of interest: None.

Financial support: None.

Ricevuto: 19 Luglio 2013; Accettato: 24 Luglio 2013

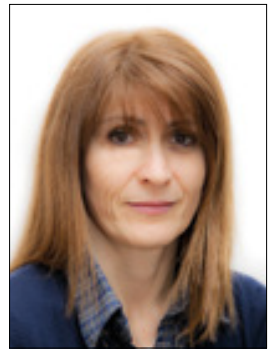

Antonella Montani

\section{Introduzione}

Le manovre di connessione e di disconnessione dei Cateteri Venosi Centrali (CVC) sono potenzialmente causa dell'ingresso, nel circolo ematico, di microrganismi che possono determinare infezioni catetere correlate (CRBSI) nei centri dialisi. Per questo e per il sempre maggiore uso dei CVC nei centri dialisi sta diventando sempre più importante affidare la gestione dei CVC a del personale specificatamente addestrato e cosciente delle gravi problematiche a questi correlate e far seguire in modo scrupoloso a tutto lo staff medico infermieristico un protocollo ben stabilito e condiviso per la gestione dei CVC. In questo articolo viene proposto il protocollo seguito nel nostro centro, elaborato e modificato anche in base al confronto con altre realtà. Abbiamo adottato questo protocollo sin dal 1993 con ottimi risultati (1).

\section{Procedura operativa della gestione dei CVC in dialisi}

Dopo un'accurata igiene delle mani, l'operatore indossa dispositivi di protezione come occhiali, mascherina e guanti non sterili. Rimuove il cerotto adesivo che ricopre la medicazione dal basso verso l'alto tenendo ben fermo il catetere.

L'operatore pulisce con garze sterili imbevute di acqua e sapone (sono disponibili in commercio soluzioni già pronte) la cute intorno al catetere, rimuovendo, così, i residui di adesivo dalla superficie cutanea, poi sciacqua e asciuga utilizzando garze sterili. L'utilizzo del sapone, per il suo potere antibatterico, serve a ottenere una pulizia accurata, oltre a permettere di sgrassare adeguatamente la cute.

A questo punto, l'operatore pulisce l'emergenza cutanea del catetere con garze sterili imbevute di soluzione fisiologica, adottando un movimento circolare dal centro verso l'esterno, e, quindi, asciuga l'emergenza cutanea sempre con garze sterili (Fig. 1).

Dopo aver applicato una piccola quantità di mupirocina pomata su una garza sterile intorno all'emergenza del catetere (se non esiste incompatibilità con il materiale di cui è fatto il CVC), l'operatore copre l'emergenza cutanea con un cerotto medicato sterile, avendo cura di premere lo stesso intorno al tratto di fuoriuscita del catetere dalla cute.

Quindi, applica un secondo cerotto tagliato a coda di rondine in senso contrario al precedente, in modo da inglobare e fissare il catetere alla cute, riducendo, così, il pericolo di un eventuale basculamento dello stesso CVC (Fig. 2).

In alternativa all'applicazione di mupirocina, viene applicato un cerotto medicato con clorexidina. Questa medicazione ha il vantaggio di avere una durata di 7 giorni e permette, pertanto, un risparmio di tempo nella fase di attacco.

\section{Inizio della seduta emodialitica}

Il paziente indossa la mascherina. Nella fase di attacco della seduta dialitica si procede con due operatori. Il primo, dopo aver indossato mascherina e guanti non sterili, avvolge le estremità terminali del CVC e delle linee del circuito extracorporeo del rene artificiale con garze sterili imbevute di amuchina al $50 \%$, che lascia riposare per 5 minuti, ovvero per il tempo d'azione indicato perché tale disinfettante agisca. In 


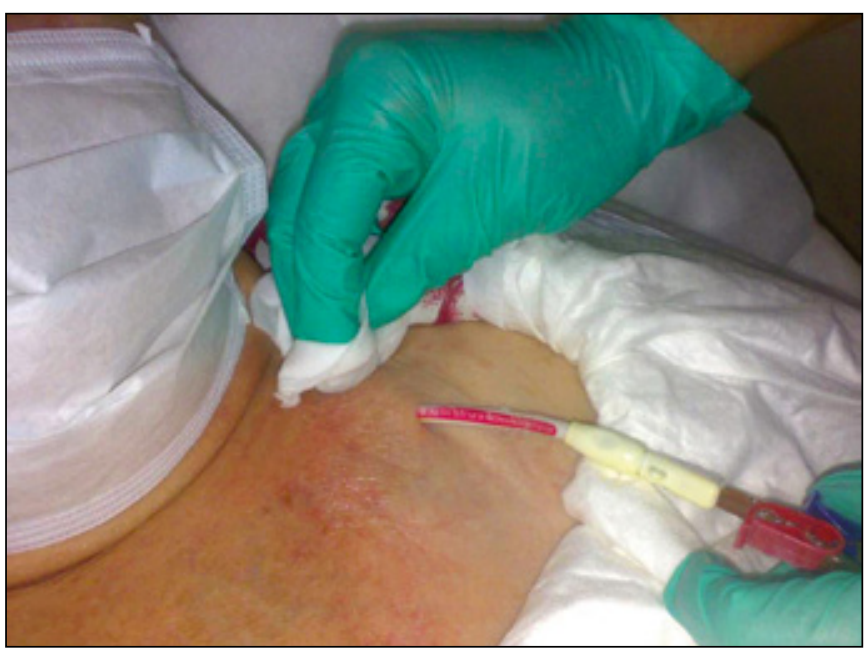

Fig. 1 - Pulizia emergenza cutanea.

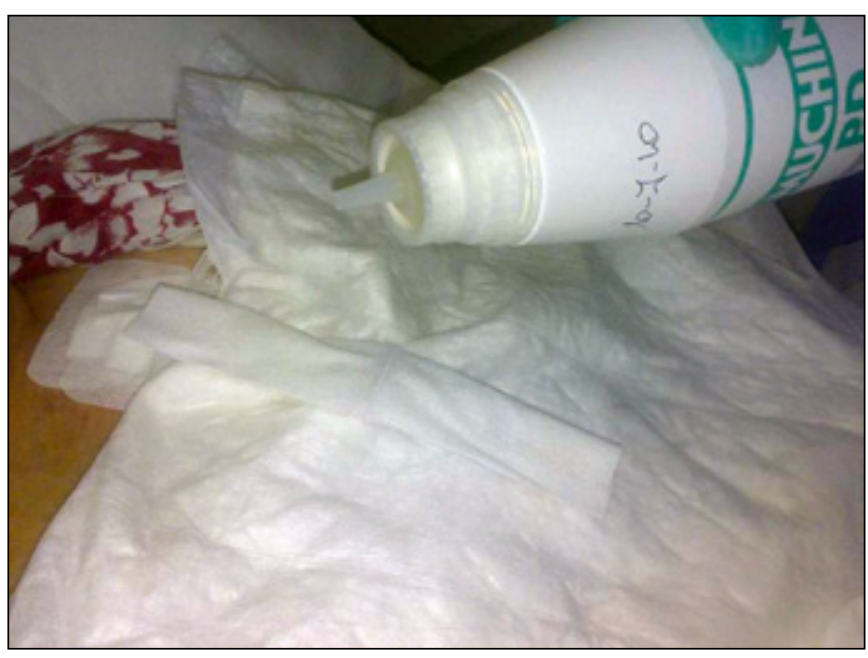

Fig. 3 - Disinfezione delle branche.

alternativa, si può usare della clorexidina al $2 \%$, con il vantaggio di ridurre i tempi di applicazione a 1 minuto (Fig. 3).

Un secondo operatore, con dispositivi di protezione e guanti sterili, posa un telino sterile sotto le estremità terminali del $\mathrm{CVC}$, mentre il primo operatore le mantiene sollevate e, quindi, le posa sul telino, sempre avendo cura di non toccare alcuna parte del campo sterile, e, poi, passa al secondo operatore una siringa sterile da $20 \mathrm{cc}$.

Il secondo operatore rimuove i tappi del CVC e aspira circa 5-6 cc di sangue dalle branche del CVC. La connessione degli estremi del CVC a quelli delle linee del circuito extracorporeo del rene artificiale avviene toccando solamente gli estremi avvolti nell'impacco con il disinfettante. A questo punto, si fissano le linee con cerotti e si chiude il telino, al fine di isolare e mantenere la maggiore pulizia possibile dei punti di connessione tra CVC e circuito extracorporeo durante tutta la seduta emodialitica (Fig. 4).

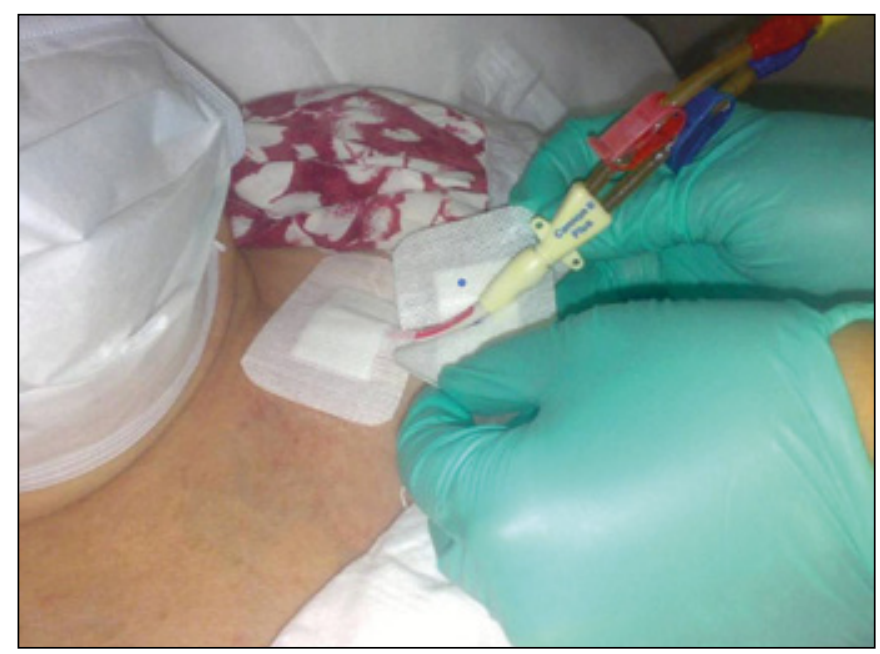

Fig. 2 - Applicazione di cerotti sopra emergenza catetere.

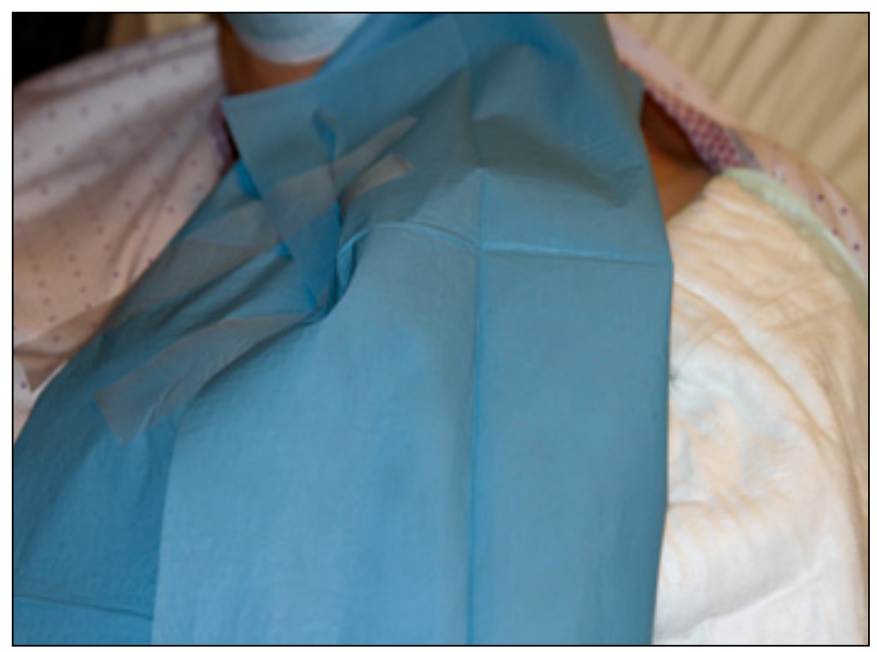

Fig. 4 - Fissaggio delle linee.

\section{Termine della seduta emodialitica}

Anche in questa fase si procede con due operatori. Al termine della seduta dialitica, il primo operatore con mascherina e guanti non sterili avvolge i terminali del $\mathrm{CVC}$ con garze imbevute di amuchina al 50\% (o clorexidina al 2\%) e lascia agire per il tempo indicato.

Il secondo operatore indossa dispositivi di protezione e guanti sterili e provvede a preparare un campo sterile così composto:

- una siringa sterile da $30 \mathrm{cc}$ riempita di soluzione fisiologica - una siringa sterile da $5 \mathrm{cc}$ riempita con soluzione lock

- due tappini di chiusura

- due garze sterili di cui una viene imbevuta di amuchina al 50\%.

Il primo operatore apre l'impacco in cui è avvolto il ter- 


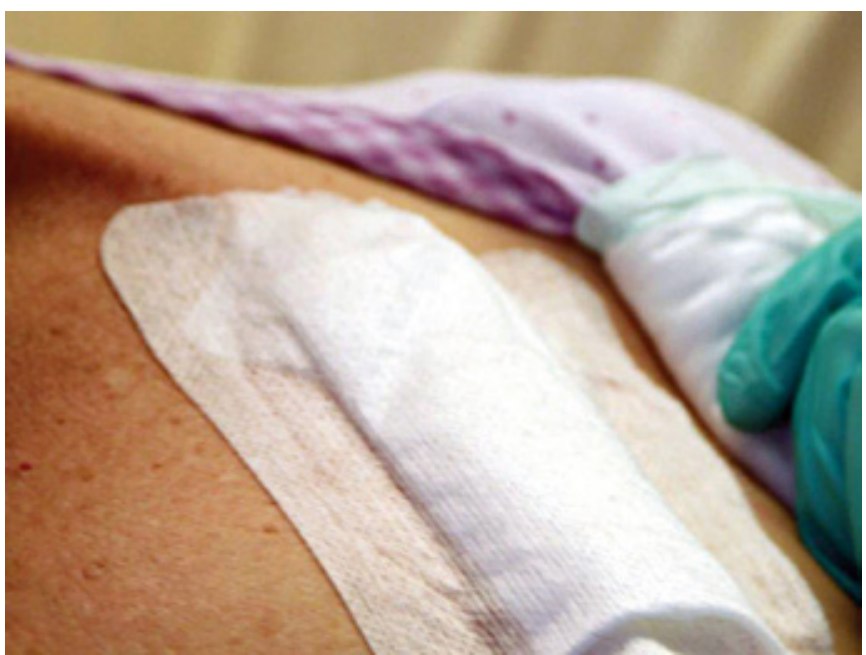

Fig. 5 - Medicazione finale.

minale del CVC.

Il secondo operatore provvede a disconnettere le linee del rene artificiale e lava i terminali del CVC con $15 \mathrm{cc}$ di soluzione fisiologica per branca. Lentamente riempie le branche con lock di chiusura con la quantità indicata sulla branca stessa. Pulisce i raccordi del CVC da eventuali residui di sangue con la garza con disinfettante e asciuga; infine, chiude con i tappini.

Al termine, uno dei due operatori avvolge i terminali del CVC con garza non sterile per evitare eventuali decubiti, ricopre con benda elastica coesiva e fissa il tutto con un cerotto a piatto (Fig. 5).

Va sottolineato, per la sua ovvia importanza, che, durante il trattamento dialitico, in qualunque caso si debba disconnettere il CVC dalle linee del circuito extracorporeo e riconnetterlo, si deve procede con le stesse modalità esposte sopra, ovvero con manovre poste in atto da due operatori, di cui uno sterile, proprio come se si trattasse di un attacco/stacco.

\section{Conclusioni}

La stretta osservanza da parte del personale medico e infermieristico del protocollo riportato sopra ha permesso, dal 1993 a oggi, di mantenere un tasso molto basso di infezioni, inferiore a 1 ogni 1000 giorni di permanenza del catetere. Questo ci ha consentito non solo di evitare eventi clinici potenzialmente gravi per i nostri pazienti, ma anche di ridurre $\mathrm{i}$ costi legati ai ricoveri associati alle infezioni e il carico di lavoro medico e infermieristico. È nostra convinzione che gli eccellenti risultati che continuiamo a ottenere non siano legati soltanto al protocollo in sé, ma soprattutto alla rigorosa adesione alla pro- cedura da parte di tutto lo staff. La combinazione di un buon protocollo con la sua costante applicazione consente di ridurre i rischi di contaminazione batterica sia nelle fasi di connessione al circuito extracorporeo per dialisi che di sconnessione, riducendo l'incidenza di infezioni correlate al CVC.

\section{Riassunto}

Dal 1993, nel nostro centro dialisi, abbiamo adottato un protocollo per la prevenzione delle infezioni CVC correlate, che proponiamo qui di seguito e grazie al quale, da 10 anni, manteniamo un tasso di infezioni inferiore a 1/1000 giorni di permanenza del catetere.

Parole chiave: Protocollo, Emodialisi, Infezioni CVC correlate (CRBSI)

Dichiarazione di conflitto di interesse: Gli Autori dichiarano di non avere conflitto di interessi.

Contributi economici agli Autori: Gli Autori dichiarano di non aver ricevuto sponsorizzazioni economiche per la preparazione dell'articolo.

Indirizzo degli Autori:

Infermiera Antonella Montani

Reparto di Dialisi

Ospedale G. Fornaroli

Via Donatore di Sangue 51

20013 Magenta (MI)

antonella.montani@ao-legnano.it

\section{Bibliografia}

1. Cornacchiari M, Heidempergher M, Guastoni C, et al. Effectiveness of a protocol for the prevention of hemodialysis venous catheter-related infections. J Vasc Access 2011; 12: 313-7. 